\title{
Cytotoxic lymphocytes in B-cell chronic lymphocytic leukemia
}

\author{
A flow cytometric study of peripheral blood, lymph nodes and bone marrow \\ L. W. M. M. Terstappen, B. G. de Grooth, I. Segers-Nolten, and J. Greve \\ University of Twente, Department of Applied Physics, Cell Characterization Group, P.O. Box 217, \\ NL-7500 AE Enschede, The Netherlands
}

Received May 30, 1989 / Accepted August 18, 1989

Summary. The occurrence of cytotoxic lymphocyte subpopulations (i.e., $\mathrm{CD} 16^{+}, \mathrm{CD} 57^{+}$and cytotoxic $\mathrm{CD} 8^{+}$) was studied in the peripheral blood of 18 B-cell chronic lymphocytic leukemia (B-CLL) patients. The absolute numbers of $\mathrm{CD} 57^{+}, \mathrm{CD} 16^{+}$and cytotoxic $\mathrm{CD}^{+}$lymphocytes were increased in the peripheral blood of untreated patients as compared with healthy donors, suggesting a causal relation with the accumulation of malignant B-cells. For 5 B-CLL patients and 5 hematological normal donors, the lymphocyte subpopulations in peripheral blood, lymph nodes and bone marrow were determined. A significant immune response was observed in the lymph nodes of the patients, as reflected by the $\mathrm{CD} 3^{+}$lymphocytes, which were $1.7-27$ times larger in the patients lymph nodes than in their peripheral blood and bone marrow. In contrast, with peripheral blood this was mainly caused by an increase in $\mathrm{CD} 4^{+}$lymphocytes. The CD 57 lymphocytes in the lymph nodes of the patients had abnormal orthogonal light-scattering signals and an abnormal density of $\mathrm{CD} 57^{+}$receptors in comparison with their peripheral blood $\mathrm{CD} 57^{+}$lymphocytes or the $\mathrm{CD} 57^{+}$lymphocytes in the peripheral blood, bone marrow and tonsils of the hematological normal donors. This study shows that although a significant increase of cytotoxic lymphocytes in the peripheral blood of B-CLL patients is observed, the actual distributions of the non-malignant lymphocytes can be quite different at the actual tumor sites, i.e., bone marrow and lymph nodes.

Key words: Cytotoxic lymphocytes - B-CLL Peripheral blood - Bone marrow - Lymph nodes

Offprint requests to: L. W. M. M. Terstappen, Becton Dickinson Monoclonal Center, Inc., 2375 Garcia Avenue, Mountain View, CA 94043, USA

\section{Introduction}

B-cell chronic lymphocytic leukemia (B-CLL) is characterized by a progressive accumulation of malignant B lymphocytes. In addition, several phenotypic and functional abnormalities of the T-lymphocyte population are described. The number of CD 4-positive $\left(\mathrm{CD}^{+}\right)$lymphocytes is decreased, whereas the number of $\mathrm{CD}$ 8-positive $\left(\mathrm{CD}^{+}\right)$lymphocytes is increased, both relative to what is found in healthy donors $[2,8,14-16,19]$. Moreover, the $\mathrm{CD} 8^{+}$lymphocytes in B-CLL patients mainly consist of cytotoxic $\mathrm{CD} 8^{+}$lymphocytes [22]. A large percentage of the $\mathrm{CD} 4^{+}$lymphocytes coexpress NK lineage markers [24]. Also, the CD 57 antigen is present on a significantly higher part of the T-lymphocytes in B-CLL patients in comparison with healthy donors [5]. Functional studies on $\mathrm{T}$ lymphocytes from B-CLL patients give contradictory results $[1,3,4,6,7,9-13,17,18]$.

As cytotoxic lymphocytes may have a destructive effect on malignant B lymphocytes, it is of importance to know the composition of the cytotoxic lymphocyte population in the different blood compartments. Therefore, we have analyzed cytotoxic lymphocyte subpopulations in the peripheral blood, bone marrow and lymph nodes of normal donors and B-CLL patients.

\section{Materials and methods \\ Patients and controls. The study included 18 patients with B-CLL, with a mean age of 60 years (range $41-85$ ). The pa- tients were graded using the Rai clinical staging system. Six pa- tients were included with Rai stage 0 with stable disease for at least 2 years. Four patients graded stage 0 were in partial remis- sion after splenic irradiation and/or chemotherapy and gluco- corticosteroid treatment. These patients were treated because of progressive disease (Rai stages III, IV) and received main- tenance treatment with chlorambucil and prednisolone. An- other six patients with progressive disease (Rai stages II, III, IV) who had previously been untreated and two patients graded Rai}


L. W. M. M. Terstappen et al.: Cytotoxic lymphocytes in B-CLL

stage IV who were refractory to chemotherapy and glucocorticosteroid treatment were included. The control group included 30 healthy individuals who were from 22 to 90 years of age. Peripheral blood and bone marrow aspirates were obtained from 5 normal adults. Tonsils obtained after tonsillectomy of hematological healthy donors were used as controls for the lymph nodes.

Preparation of lymphocytes. Peripheral blood was collected by venipuncture into vacutainer tubes containing heparin as anticoagulant (150 USP IU sodium heparin $/ 10 \mathrm{ml}$; Venoject Terumo Europe). The absolute number of peripheral blood lymphocytes was determined with a H6000 (Technicon, Tarrytown, NY). The absolute number of lymphocyte subpopulations was obtained using this number. Bone marrow aspirates were collected using heparin as anticoagulant. Lymphocytes were obtained after density separation (density $1,077 \mathrm{~g} / \mathrm{cm}^{3}$, Percoll Pharmacia Fine Chemicals, Uppsala, Sweden) [20]. Four cervical lymph nodes and one lymph node from the groin were removed surgically under local anesthesia. The lymph nodes varied in diameter from $2-5 \mathrm{~cm}$. The lymph nodes and tonsils were put in a solution of minimal essential medium (MEM, Flow Laboratories, Irvine, Scotland) containing $10 \%$ fetal calf serum (FCS, GIBCO, Paisley, Scotland) and $10^{6}$ IU Penicillin $\mathrm{G} / \mathrm{L}$ (Pen). The lymph nodes and tonsils were cut on a polyester mesh (pore diameter $30 \mu \mathrm{m}$, Visprox BV, Haarlem, The Netherlands), using scissors and tweezers. During preparation, the lymph nodes were continuously rinsed with MEM containing FCS and Pen. The cell suspension was washed 3 times with MEM at $200 \mathrm{~g}$ for $5 \mathrm{~min}$. For each patient peripheral blood, bone marrow and lymph nodes were collected and processed on the same day. The lymphocyte suspensions were adjusted to a concentration of $1.10^{7}$ lymphocytes $/ \mathrm{ml}$ with phosphate-buffered saline containing $0.05 \%$ sodium azide and $1 \%$ bovine serum albumin (PBS).

Lymphocyte subset identification. Lymphocytes were incubated with an optimal concentration of fluorescently labeled monoclonal antibodies. The labeled lymphocytes were resuspended in PBS to a final concentration of $10^{6}$ lymphocytes $/ \mathrm{ml}$. The following monoclonal antibodies were used: CD 3 (anti-Leu 4 FITC, PE), CD 4 (anti-Leu 3 FITC), CD 8 (anti-Leu 2a FITC, PE), CD 16 (anti-Leu 11a FITC) and CD 57 (anti-Leu 7 FITC). The Mabs were purchased from Becton Dickinson (San Jose, Calif.). Flow cytometric analysis was performed within $8 \mathrm{~h}$ after sample collection to preserve both the optimal binding of monoclonal antibody and light-scattering properties of lymphocytes. The flow cytometer (FCM) used is described in detail elsewhere [20]. By adjustment of the FCM with standard microspheres, reproducible apparatus conditions were obtained for the measurement of light scattering and fluorescence intensity [21]. Residual monocytes, contaminating the lymphocyte preparation were excluded from the measured data by discrimination on forward and orthogonal light scattering in the FCM [21]. Discrimination levels of fluorescence staining were chosen, so that less than $0.02 \%$ of the cells of a control sample fell above the level.

Orthogonal light-scatter histograms of the $\mathrm{CD} 8$-positive $\left(\mathrm{CD}^{+}\right)$lymphocytes were obtained after gating on the fluorescence intensity of the immunofluorescent-labeled cells. The $\mathrm{CD} 8^{+}$lymphocytes were divided into suppressor $\left(\mathrm{CD} 8 \mathrm{a}^{+}\right)$lymphocytes and cytotoxic $\left(\mathrm{CD}^{\circ} \mathrm{b}^{+}\right)$lymphocytes by dividing the orthogonal light scatter histogram of $\mathrm{CD} 8^{+}$lymphocytes into two components [20]. CD $8 \mathrm{a}^{+}$lymphocytes have a low orthogonal light-scattering signal whereas $\mathrm{CD} 8 \mathrm{~b}^{+}$lymphocytes have a high orthogonal light-scattering signal [20]. Each measurement contained at least 40,000 lymphocytes.

\section{Results}

\section{Lymphocyte subpopulations in peripheral blood}

The absolute numbers of lymphocytes positive with the monoclonal antibodies, CD 57 and CD 16 were determined in the peripheral blood of $18 \mathrm{~B}-\mathrm{CLL}$ patients and 30 healthy donors. In addition, the absolute numbers of $\mathrm{CD} 8^{+}$cytotoxic lymphocytes $\left(\mathrm{CD} 8 \mathrm{~b}^{+}\right)$were determined as described in Materials and methods. The absolute number of $\mathrm{CD} 8 \mathrm{~b}^{+}$, $\mathrm{CD} 57^{+}$and $\mathrm{CD} 16^{+}$lymphocytes of B-CLL patients were significantly different from those of healthy controls: $P<0.0001, P<0.00001$ and $P<0.000001$, respectively (Kruskal Wallis test). In Fig. 1 the absolute numbers of the three lymphocyte subpopulations are given. The absolute number of cytotoxic lymphocyte populations in the B-CLL patients has a broad distribution, as compared with healthy donors. The total lymphocyte count of the B-CLL
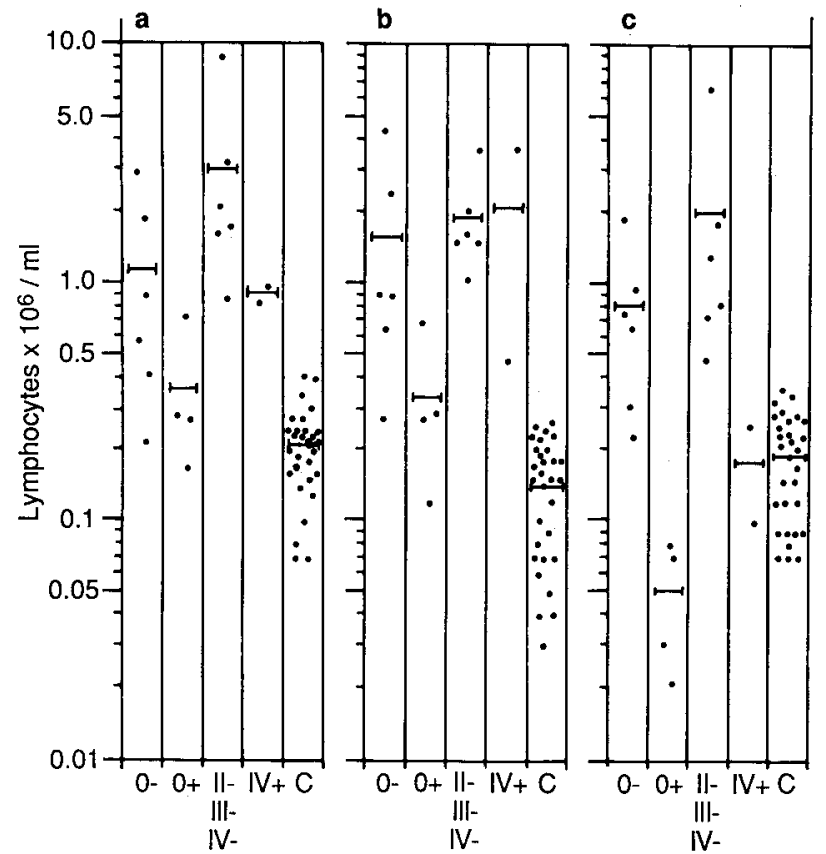

Fig.1 a-c. Absolute number of $\mathrm{CD} 8 \mathrm{~b}^{+}$(a), $\mathrm{CD} 57^{+}$(b) and CD $16^{+}$lymphocytes (c) in the peripheral blood of $18 \mathrm{~B}-\mathrm{CLL}$ patients subdivided by Rai clinical stage; stage $0-$ ( - no treatment), $0+(+$ on therapy), II - , III - , IV,- IV + and 30 healthy controls (c). Numbers are given on a logarithmic scale and mean values are indicated as horizontal bars in the figures. Statistical evaluation of the data according to the two-sided Wilcoxon test showed significant differences between the results obtained for the following groups: a $0-$ and controls $(P<0.002)$; II - , III - , IV- and controls $(P<0.0002) ; 0+$ and II - , III - , IV$(P<0.01)$ b $0-$ and controls $(P<0.0002)$; II - , III - , IVand controls $(P<0.0002) ; 0+$ and controls $(P<0.03) ; 0+$ and II - , III - , IV $-(P<0.01)$ c $0-$ and controls $(P<0.02)$; $0+$ and II - , III - , IV- $(P<0.0002) ; 0-$ and $0+(P<0.01)$; II - , III - , IV - and $0+(P<0.01)$ 
patients did not correlate significantly with the absolute number of $\mathrm{CD} 8 \mathrm{~b}^{+}, \mathrm{CD} 57^{+}$and $\mathrm{CD} 16^{+}$lymphocytes $\left(r^{2}=0.35,0.24\right.$ and 0.34 , respectively).

Subdivision into different disease stages revealed that untreated patients with progressive disease had an increased absolute number of $\mathrm{CD} 8 \mathrm{~b}^{+}, \mathrm{CD} 57^{+}$ and $\mathrm{CD} 16^{+}$lymphocytes as compared with healthy donors. Untreated patients with benign disease showed normal or increased absolute numbers of cytotoxic lymphocytes for the three subpopulations. A remarkable observation is that treated patients, graded Rai stage 0 , had decreased absolute numbers of ${\mathrm{CD} 16^{+}}^{+}$lymphocytes, whereas the number of $\mathrm{CD} 8 \mathrm{~b}^{+}$and $\mathrm{CD} 57^{+}$lymphocytes were within the normal range for these patients.

\section{Lymphocyte subpopulations in the bone marrow and lymph nodes}

The percentage of cells positive with the monoclonal antibodies CD 3, CD 4, CD 8, CD 57 and CD 16 were determined in the peripheral blood, bone marrow and lymph nodes of five patients graded Rai stages II, III and IV and five controls. Whereas the results obtained from peripheral blood and bone marrow could be compared with those found in normal donors, no normal lymph node material could be obtained from these donors. To obtain an indication of the distribution of lymphocyte subpopulations in lymphoid tissue, we chose tonsils as the control material for the patients' lymph nodes. The data are presented in Table 1. Compared to the control group, the percentages of lymphocyte subpopulations in the peripheral blood and bone marrow of the patients are considerably lower due to the overwhelming number of malignant $B$ cells. In contrast, in the peripheral blood and bone marrow, the percentage of $\mathrm{CD} 3^{+}$lymphocytes was remarkably large in the lymph nodes of the patients. A large variation in the percentage of $\mathrm{CD} 3^{+}$lymphocytes was found in the tonsils; the remaining cells were primarily $\mathrm{B}$ lymphocytes $\left(\mathrm{CD} 19^{+}\right)$. Interestingly, in the patients' lymph nodes, all the $\mathrm{CD}^{+}$lymphocytes could be accounted for by $\mathrm{CD} 4^{+}$and $\mathrm{CD} 8^{+}$ lymphocytes, which was not the case for the $\mathrm{CD} 3^{+}$ lymphocytes in the tonsils. For a more detailed analysis of the non-malignant cells, the $\mathrm{CD} 4^{+}, \mathrm{CD} 8^{+}$, $\mathrm{CD} 57^{+}$and $\mathrm{CD} 16^{+}$cells were normalized on the $\mathrm{CD}^{+}$lymphocytes in the same blood compartment. The results are shown Fig. $2 \mathrm{a}-\mathrm{d}$, respectively. The results from the tonsils were obtained from different donors and are shown in a separate column. Furthermore, the mean ratios and standard deviation of the peripheral blood cell populations of 30 healthy donors are given in a separate column.

In all patients, the CD $4 / C D 3$ ratio was larger in lymph nodes in comparison with the peripheral blood. In contrast, CD 4/CD 3 ratios were clearly lower in tonsils as compared with peripheral blood CD 4/CD 3 ratios in healthy donors. A large variation in the CD 4/CD 3 ratio was found in the bonemarrow aspirates of patients and controls.

No difference was found between the peripheral blood $\mathrm{CD} 8 / \mathrm{CD} 3$ ratio of patients and controls. In the patients, the bone-marrow $\mathrm{CD} 8 / \mathrm{CD} 3$ ratios were lower than peripheral blood $\mathrm{CD} 8 / \mathrm{CD} 3$ ratios, whereas the opposite was observed for the control group. The CD 8/CD 3 ratios of the patients' lymph nodes were undoubtedly lower than the peripheral blood and bone-marrow CD 8/CD 3 ratios, but within the range found in the tonsils.

Table 1. Percentage of $\mathrm{CD} 3^{+}, \mathrm{CD} 4^{+}, \mathrm{CD} 8^{+}, \mathrm{CD} 57^{+}$and $\mathrm{CD} 16^{+}$lymphocytes in the peripheral blood, bone marrow and lymph nodes of five B-CLL patients and five normal donors. Note that the tonsils do not originate from the same donors as the peripheral blood and bone marrow

\begin{tabular}{|c|c|c|c|c|c|c|c|c|c|c|c|c|c|c|c|c|}
\hline \multirow[t]{2}{*}{$P$} & \multirow[t]{2}{*}{ ST } & \multicolumn{5}{|c|}{ Peripheral blood } & \multicolumn{5}{|c|}{ Bone marrow } & \multicolumn{5}{|c|}{ Lymph node } \\
\hline & & $\mathrm{CD} 3$ & $\mathrm{CD} 4$ & CD 8 & CD 57 & CD 16 & CD 3 & $\mathrm{CD} 4$ & CD 8 & CD 57 & CD 16 & CD 3 & $\mathrm{CD} 4$ & $\mathrm{CD} 8$ & CD 57 & CD 16 \\
\hline 1 & $\mathrm{IV}^{\mathrm{a}}$ & 1.5 & 0.7 & 0.5 & 1.1 & 0.4 & 2.0 & 1.2 & 0.6 & 1.0 & 0.2 & 2.5 & 1.7 & 0.3 & 1.0 & 1.0 \\
\hline 2 & III & 1.5 & 0.7 & 0.7 & 0.4 & 0.5 & 2.2 & 1.0 & 0.8 & 0.4 & 0.4 & 44.5 & 40.8 & 5.7 & 1.0 & 0.2 \\
\hline 3 & II & 6.5 & 3.1 & 2.5 & 2.3 & 0.7 & 4.8 & 3.9 & 1.6 & 1.9 & 0.3 & 11.4 & 8.3 & 3.2 & 2.3 & 0.0 \\
\hline 4 & III & 3.6 & 2.0 & 1.3 & 0.6 & 0.7 & n.d. & n.d. & n.d. & n.d. & n.d. & 14.9 & 9.4 & 2.4 & 0.7 & 0.2 \\
\hline 5 & IV & 2.2 & 1.5 & 0.7 & 2.1 & 0.2 & n.d. & n.d. & n.d. & n.d. & n.d. & 25.9 & 18.3 & 5.7 & 5.9 & 0.8 \\
\hline $\mathrm{C}$ & & & & & & & & & & & & \multicolumn{5}{|c|}{ Tonsils } \\
\hline 1 & & 80.6 & 60.0 & 23.7 & 3.2 & 4.9 & 54.1 & 44.7 & 23.7 & 0.8 & 2.1 & 52.8 & 22.8 & 5.4 & 1.5 & 0.4 \\
\hline 2 & & 62.1 & 41.7 & 32.6 & 7.6 & 6.8 & 28.4 & 13.4 & 17.6 & 1.0 & 1.0 & 80.2 & 42.8 & 5.2 & 1.8 & 0.6 \\
\hline 3 & & 64.6 & 41.5 & 27.0 & 2.3 & 4.0 & 24.5 & 20.5 & 22.6 & 0.2 & 0.7 & 6.9 & 3.8 & 0.9 & 0.5 & 0.0 \\
\hline 4 & & 65.1 & 46.6 & 26.4 & 9.7 & 2.7 & 26.7 & 15.4 & 20.0 & 3.0 & 0.2 & 25.0 & 11.1 & 2.6 & 0.8 & 0.0 \\
\hline 5 & & 72.3 & 47.9 & 26.9 & 4.8 & 8.4 & 45.3 & 29.5 & 24.0 & 2.9 & 0.9 & 23.6 & 10.7 & 4.8 & 1.4 & 0.1 \\
\hline
\end{tabular}

a On treatment; P, patient; C, control; ST, Rai stage 

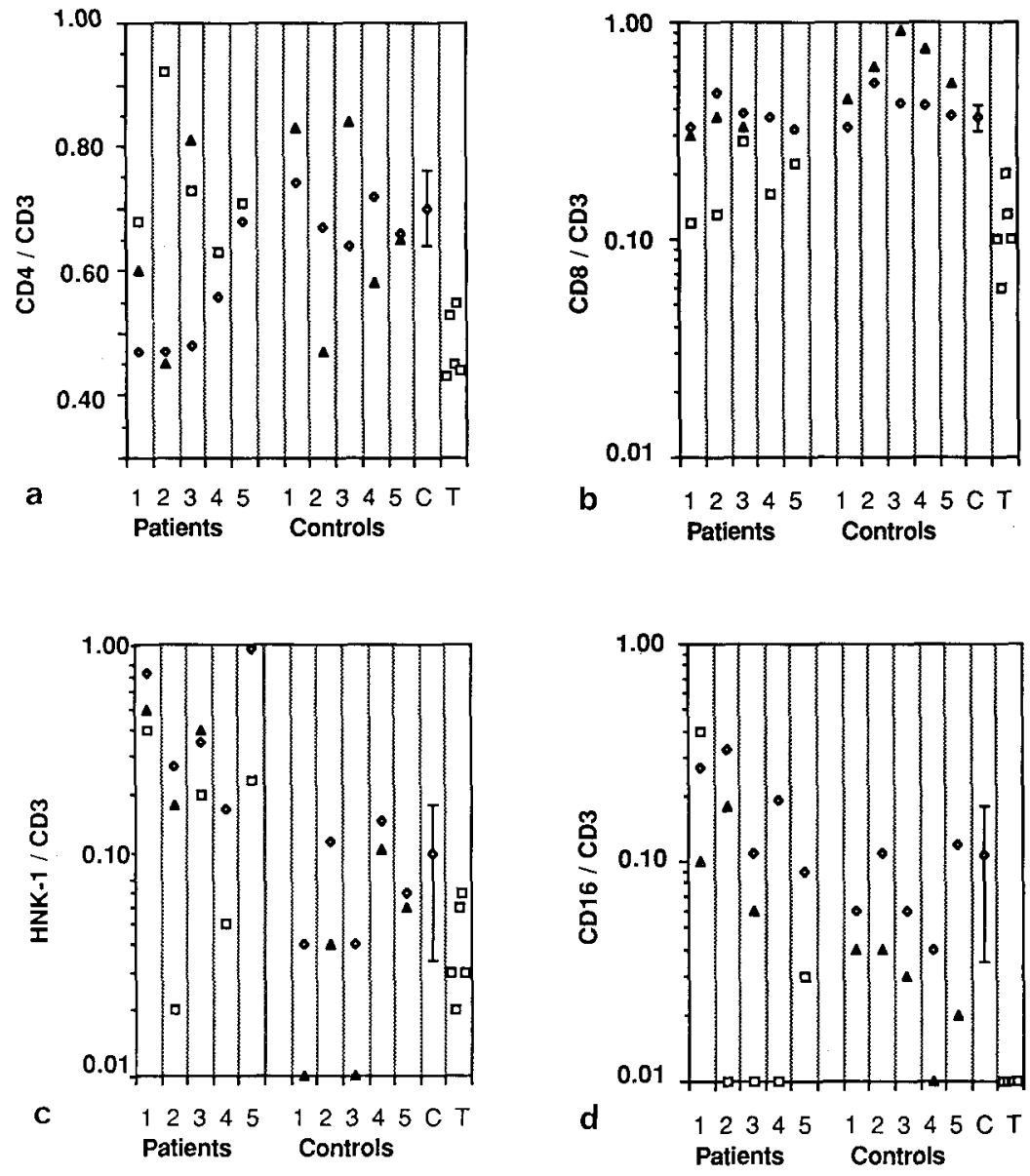

Fig. $2 \mathrm{a}-$ d. Relative occurrence of $\mathrm{CD}^{+}(\mathbf{a})$, $\mathrm{CD} 8^{+}(\mathbf{b})$, HNK-1 $\left(\mathrm{CD} 57^{+}\right)$(c) and CD $16^{+}$ lymphocytes (d) in the peripheral blood $(\diamond)$, bone marrow $(\boldsymbol{A})$ and lymph nodes $(\square)$ of 5 B-CLL patients (1, Rai stage IV; 2, Rai stage III; 3, Rai stage II; 4, Rai stage III; 5, Rai stage IV, on treatment) and 5 healthy controls. The results obtained from five tonsils are indicated in a separate column $(T)$ as are the mean and standard deviation of the peripheral blood of 30 healthy donors $(C)$. Data were normalized based on the percentage of $\mathrm{CD}^{+}$lymphocytes in the peripheral blood, bone marrow or lymph node. Note the linear scale of the CD 4/CD 3 versus the logarithmic scale of $\mathrm{CD} 8 / \mathrm{CD} 3, \mathrm{HNK}-1 / \mathrm{CD} 3$ and CD $16 / \mathrm{CD} 3$
Both peripheral blood and bone-marrow CD 57/CD 3 ratios were markedly larger in patients compared with the control group. Heterogeneous results were obtained for $\mathrm{CD} 57 / \mathrm{CD} 3$ ratios in the lymph nodes of the patients. In two cases the $\mathrm{CD} 57 / \mathrm{CD} 3$ ratios were within the range found in tonsils whereas, in the other three cases the CD 57/CD 3 ratios were eminently larger.

In both patients and the control group, the CD 16/CD 3 ratios were lower in bone marrow compared with peripheral blood. In tonsils, CD $16^{+}$was not present or was just above the detection level. In two of the patients the CD 16/CD 3 ratios in lymph nodes were clearly increased; note that both patients also had an enlarged CD 57/CD 3 ratio.

\section{Heterogeneity within the CD57+ lymphocytes}

Large differences were observed within the CD $57^{+}$ lymphocyte population in the different blood compartments of the B-CLL patients. Apparently, different subpopulations were present within the $\mathrm{CD} 57^{+}$population. The relative occurrence of these subpopulations differed between the specific blood compartments. This is illustrated for one patient in Fig. 3 where two-parameter density maps of orthogonal light scattering and FITC-conjugated CD 57 fluorescence intensity are presented for lymphocytes obtained from the peripheral blood (Fig. 3a), bone marrow (Fig. 3 b), and lymph node (Fig. $3 \mathrm{c}$ ). Due to the enormous amount of malignant B-cells in B-CLL patients the majority of lymphocytes did not express the CD 57 antigen. The malignant Blymphocytes had low orthogonal light-scattering signals, a property of normal B lymphocytes [21] and the lymphocytes of B-CLL patients [23]. In peripheral blood, the $\mathrm{CD} 57^{+}$lymphocytes showed a high density of the CD 57 antigen (i.e., a high fluorescence intensity) and a high orthogonal light scattering. Completely different characteristics of the CD $57^{+}$lymphocytes were observed (Fig. $3 \mathrm{c}$ ) in the lymph nodes of this patient. Here the CD $57^{+}$lymphocytes had a low density of the CD 57 antigen and a low orthogonal light scattering. In the bone marrow of this patient both $\mathrm{CD} 57^{+}$lymphocytes populations could be observed (Fig. 3 b). In all patients we found a similar heterogeneity of the 

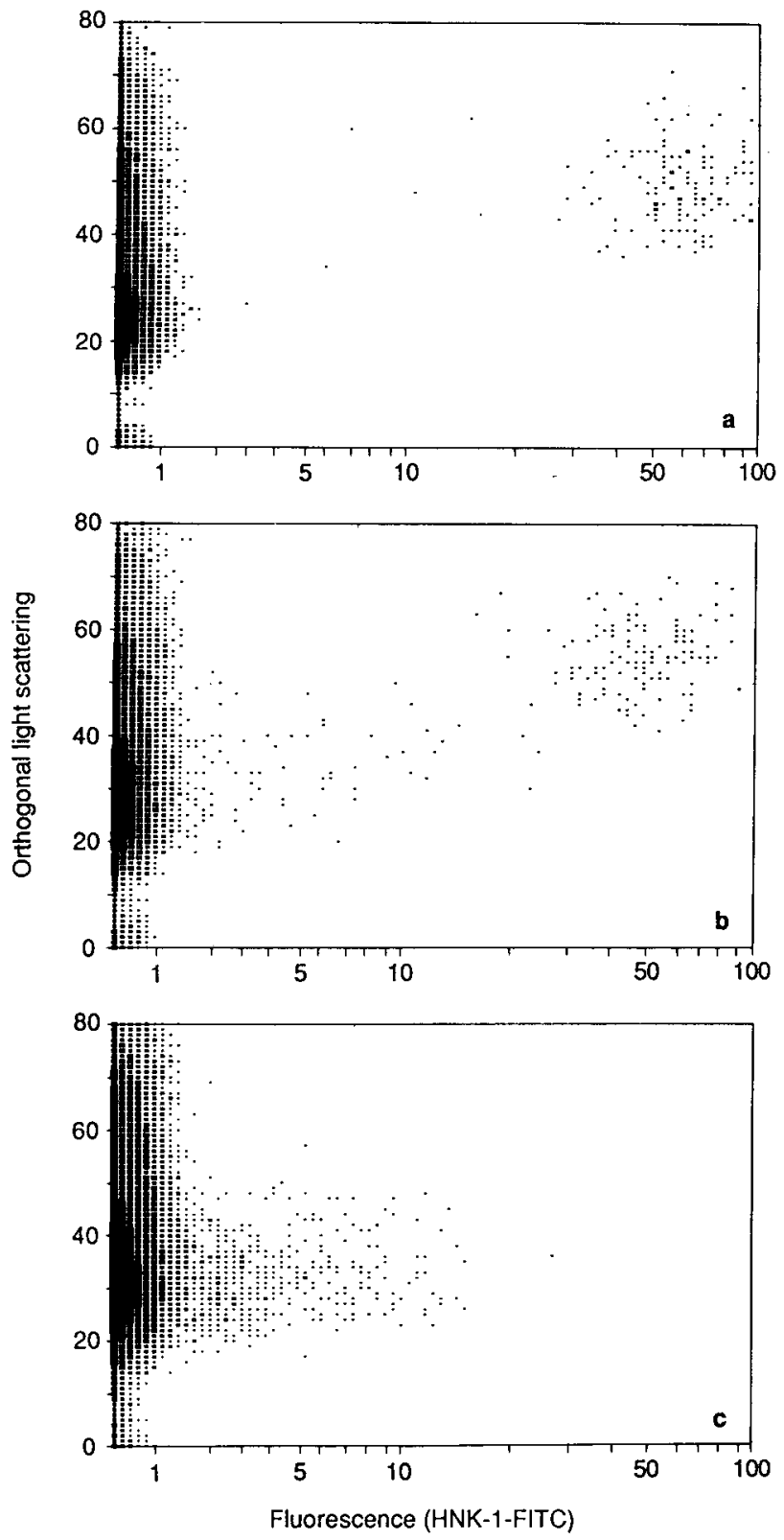

Fig. $3 \mathrm{a}-$ c. Two density parameter maps of orthogonal light scattering (ordinate) and fluorescence intensity (abscissa) of the lymphocytes of one B-CLL patient incubated with FITCconjugated HNK-1 (CD 57). Lymphocytes from the peripheral blood $\mathbf{a}$, bone marrow $\mathbf{b}$ and lymph node $\mathbf{c}$

$\mathrm{CD} 57^{+}$lymphocyte population. In contrast, the $\mathrm{CD} 57^{+}$lymphocytes in normal bone marrow aspirates and tonsils all had a high orthogonal light scattering and a high fluorescence intensity.

\section{Discussion}

Decreased CD 4/CD 8 ratios have often been found for B-CLL patients $[2,8,14-16,19]$. We have shown in a previous study that this is due to an increased number of $\mathrm{CD}^{+}$cytotoxic lymphocytes [22]. A hypothesis we have put forward is that in B-CLL patients the cytotoxic lymphocytes are involved in an active resistance of the immune system against malignant B lymphocytes. The present study was therefore primarily concerned with an analysis of lymphocyte subsets, expressing cytotoxic surface markers, in the peripheral blood, bone marrow and lymph nodes of B-CLL patients. To be significant in the defense against leukemic cells, cytotoxic lymphocytes have to be present at tumor sites such as bone marrow and the lymph nodes involved of the patients.

In this study, we show that in the peripheral blood of untreated B-CLL patients, the absolute numbers of cytotoxic lymphocyte subpopulations (i.e., $\mathrm{CD} 8 \mathrm{~b}^{+}, \mathrm{CD} 16^{+}, \mathrm{CD} 57^{+}$) were significantly increased (Fig. $1 \mathrm{a}-\mathrm{c}$ ) compared with healthy donors. By means of two-color immunofluorescence it was shown that the majority of the cytotoxic lymphocytes showed relatively high orthogonal light scattering indicative of cytoplasmatic granularity and coexpressed CD 3, CD 8, CD 16 and CD 57. The latter implies that the majority of cells involved do not belong to the "classic" NK cells. The increase in absolute numbers of cytotoxic lymphocyte subpopulations was less pronounced in patients with stable disease. In successfully treated patients, however, the absolute numbers of cytotoxic lymphocytes were within the normal range for $\mathrm{CD} 57^{+}$and cytotoxic $\mathrm{CD} 8 \mathrm{~b}^{+}$lymphocytes and were even decreased for $\mathrm{CD} 16^{+}$cells. Whether this is caused by the cytotoxic agents used in the therapy or by the reduction of tumor mass is of interest but not known.

Our observation that the absolute number of cytotoxic lymphocytes was increased in B-CLL patients indicates that, in principle, there is a great potential for active cytotoxic function in the peripheral blood of B-CLL patients. In addition, we observed a strong reaction of the immune system, especially in the lymph nodes. This was revealed by a relative increase in the $\mathrm{CD} 3^{+}$lymphocytes in the enlarged lymph nodes in comparison with the peripheral blood or bone marrow (Table 1).

The composition of the non-malignant lymphocyte populations in the lymph nodes, however, was quite different compared with the peripheral blood. Surprisingly, the dominant population in the lymph nodes was $\mathrm{CD} 4^{+}$lymphocytes (Fig. 2). In fact, the CD 4/CD 3, CD 8/CD 3, CD 57/CD 3 and CD 16/ CD 3 ratios in the lymph nodes were all inverted when compared with the peripheral blood (Fig. 2).

The relative number of $\mathrm{CD} 4^{+}$lymphocytes in the lymph nodes was also increased when compared 
with the normal values found in tonsils. Knowledge of the actual function of the $\mathrm{CD} 4^{+}$lymphocytes is of considerable interest since they could be involved in stimulating the proliferation of malignant cells or actually "helping" to defend the system against the malignant cells.

In the tonsils and in three of the patients no CD $16^{+}$cells could be detected. The CD $8 / C D 3$ ratios in the lymph nodes were within the $\mathrm{CD} 8$ / CD 3 range found in the controls. The CD 57/CD 3 ratios in lymph nodes were still larger in three of the five patients compared with the controls (Fig. 2).

A further characterization of the $\mathrm{CD} 57^{+}$lymphocytes showed the existence of two populations of $\mathrm{CD} 57^{+}$lymphocytes in the B-CLL patients. As in normal donors, the peripheral blood $\mathrm{CD} 57^{+}$lymphocytes have a high density of CD 57 antigen and a high orthogonal light-scattering signal [21]. The same characteristics were found for the CD $57^{+} \mathrm{lym}-$ phocytes as found in the tonsils and bone marrow of the controls. In contrast, the $\mathrm{CD} 57^{+}$lymphocytes found in the lymph nodes of all patients had a low density of CD 57 receptors and a low intensity of orthogonal light scattering. In the bone marrow of the patients, both normal and abnormal populations were observed. Whether these observations could be explained by the existence of two distinct populations of $\mathrm{CD} 57^{+}$lymphocytes or reflect the different maturational stages of $\mathrm{CD} 57^{+}$lymphocytes is unknown.

The absolute increase in $\mathrm{CD} 8 \mathrm{~b}^{+}, \mathrm{CD} 57^{+}$and CD $16^{+}$lymphocytes in the peripheral blood of B-CLL patients suggests a causal relation with the accumulation of malignant B lymphocytes. That these cytotoxic lymphocyte populations are responsible for the defense against malignant B lymphocytes is an oversimplification. The majority of lymphocytes involved in the enormous immune response observed at the actual tumor sites, i.e., bone marrow and especially lymph nodes, expressed the $\mathrm{CD} 4$ antigen, but not the CD 8, CD 57 or CD 16 antigens. Furthermore, the $\mathrm{CD} 57^{+}$lymphocytes in the lymph nodes of the patients were found to be abnormal. Although major changes in the lymphocyte populations can be found in the different blood compartments of B-CLL patients compared with normal donors, to actually understand the immune response evoked by the malignant cells will require studies of the function and interaction of the different lymphocyte populations at the tumor sites.

One important observation that we made when studying the different blood compartments in both normal and patients is that the relative occurrence of cell subpopulations in the peripheral blood does not reflect the actual situation in the bone marrow and lymph nodes. Moreover, one must determine the occurrence of the lymphocyte populations at the site of interest to obtain the local distribution of the lymphocyte populations. Furthermore, one must realize that the absolute number of lymphocytes in peripheral blood is only a small fraction of the total amount of lymphocytes. A small change in the amount of a lymphocyte subpopulation in a major blood compartment can cause a significant change in the concentrations of these lymphocytes in the peripheral blood.

Acknowledgements. The authors would like to express their gratitude to W. van Berkel, D. Johnson and N. Gadol for providing us with both normal and patient samples.

\section{References}

1. Bloem AC, Clevers JC, Bast EJEG, Balliex RE (1986) T-cells in B-cell chronic lymphocytic leukemia. 1. Decreased frequency of $\mathrm{T}$ lymphocytes secreting suppressor factor. Clin Exp Immunol 63: 188-193

2. Catovsky D, Lauria F, Matutes E, Foa R, Mantovani V, Tura S, Galton GAG (1981) Increase in T lymphocytes in B cell chronic lymphocytic leukemia. Br J Haematol 47: $539-544$

3. Chiorazzi N, Fu SM, Montazer G, Kunkel HG, Rai K, Gee $\mathrm{T}$ (1979) T-cell helper defect in patients with chronic lymphocytic leukemia. J Immunol 122: 1087-1090

4. Davis S (1981) Characterization of the phytohemagglutinin induced proliferating lymphocyte subpopulations in chronic lymphocytic leukemia patients using a clonogenic agar technique and monoclonal antibodies. Blood 58: 1053-1055

5. Foa R, Lauria F, Lusso P, Biubellino MC, Fierro MT, Ferrando ML, Raspadori D, Matera L (1984) Discrepancy between phenotypic and functional features of natural killer T-lymphocytes in B-cell chronic lymphocytic leukemia. Br J Haematol 58: 509-516

6. Foa R, Fierro MT, Lusso P, Raspadori D, Ferrando ML, Matera L, Malavasi F, Lauria F (1986) Reduced natural killer T-cells in B-cell chronic lymphocytic leukemia identified by three monoclonal antibodies: Leu11, A10, AB8.28. Br J Haematol 62: 151-154

7. Han T, Bloom ML, Dadey B, Bennett G, Minowada J, Sandberg AA, Ozer H (1982) Lack of autologous mixed lymphocyte reaction in patients with chronic lymphocytic leukemia: evidence for autoreactive T-cell dysfunction not correlated with phenotype, karyotype, or clinical status. Blood 60: $1075-1081$

8. Hermann F, Lochner A, Philippen H, Jauer B, Ruhl H (1982) Imbalance of T-cell subpopulations in patients with chronic lymphocytic leukemia of the B cell type. Clin Exp Immunol 49: 157-162

9. Hokland VP, Ellegaard J (1981) Immunological studies in chronic lymphocytic leukemia: II. Natural killer and antibody-dependent cellular cytotoxicity potentials of malignant and nonmalignant lymphocytes subsets and the effect of alpha-interferon. Leuk Res 5: 349-355

10. Inoshita T, Whiteside TL (1981) Imbalance of T-cell subpopulations does not result in defective helper function in chronic lymphocytic leukemia. Cancer 48: 1754-1760

11. Kay NE (1981) Abnormal T-cell subpopulation function in CLL: excessive suppressor (T8) and deficient (T4) activity with respect to B-cell proliferation. Blood 57: 418-420 
12. Lauria F, Foa R, Mantovani V, Fierra MT, Catovsky D, Tura $\mathrm{S}$ (1983) T-cell functional abnormality in B-chronic lymphocyte leukemia: evidence of a defect of the T-helper subset. Br J Haematol 54: 277-283

13. Lauria F, Raspadori D, Tura D (1984) Effect of a thymic factor on T-lymphocytes in B-cell chronic lymphocyte leukemia: in vitro and in vivo studies. Blood 164: 667-671

14. Markey GM, Alexander HD, Agnew AND, McConnell RE Morris TCM, Robbertson JH, Crockard AD, Bridges JM (1986) Enumeration of absolute numbers of T lymphocytes subsets in B-chronic lymphocytic leukemia using an immunoperoxidase technique: Relation to clinical stage. $\mathrm{Br} \mathbf{J}$ Haematol 62: 257-273

15. Mills KHG, Cawley JC (1982) Suppressor T-cells in B-cell chronic lymphocytic leukemia: relationship to clinical stage. Leuk Res 6: 653-657

16. Mittellman A, Denny T, Gebhard C, Cirrincione C, Kurland E, Kozinek B (1986) Analysis of T cell subsets in B cell chronic lymphocyte leukemia: a correlation with the stage of disease. Am J Hematol 16: 67-73

17. Mon MA de, Casas J, Laguna R, Toribic ML, Landazuri MO de, Durantiz A (1986) Lymphokine induction of NKlike cytotoxicity in T cells from B-CLL. Blood 67: 228-232

18. Platsoucas CD, Fernandes G, Gupta SL, Kempin S, Clarkson B, Good RA, Gupta S (1980) Defective spontaneous and antibody dependent cytotoxicity mediated by E-rosettepositive and E-rosette-negative cells in untreated patients with chronic lymphocytic leukemia: augmentation by in vitro treatment with interferon. J Immunol 125: 1216-1223

19. Platsoucas CD, Galinski M, Kempin S, Clarkson B, Good RA (1982) Abnormal T-lymphocyte subpopulations in patients with B-cell chronic lymphocytes leukemia: an analysis by monoclonal antibodies. J Immunol 129: 2305-2313

20. Terstappen LWMM, Grooth BG de, Nolten GMJ, Napel CHH ten, Berkel W van, Greve J (1986) Physical discrimination between human T-lymphocyte subpopulations by means of light scattering, revealing two populations of T8-positive cells. Cytometry 7: 178-183

21. Terstappen LWMM, Grooth BG de, Napel $\mathrm{CHH}$ ten, Berkel W van, Greve J (1986) Discrimination of human cytotoxic lymphocytes from regulatory and B-lymphocytes by orthogonal light scattering. J Immunol Methods 95: 211-216

22. Terstappen LWMM, Grooth BG de, Berkel W van, Napel CHH ten, Greve J (1988) Abnormal distribution of CD8 subpopulations in B-chronic lymphocytic leukemia identified by flow cytometry. Leuk Res 12: 551-557

23. Terstappen LWMM, Grooth BG de, Napel CHH ten, Berkel W van, Greve J (1988) Flow cytometric characterization of chronic lymphocytic leukemias using orthogonal light scattering and quantitative immunofluorescence. Blut 56: $201-208$

24. Verlardi A, Prehal JT, Prasthofer EF, Grossi CE (1985) Expression of NK lineage markers on peripheral blood lymphocytes with T-helper (Leu3 $+/ \mathrm{T} 4+$ ) phenotype in B cell chronic lymphocytic leukemia. Blood 65: 149-155

25. Wolos JA, Davey FR (1980) Function of lymphocyte subpopulations in chronic lymphocytic leukemia. Activity in the allogenic and autologous mixed lymphocyte reaction. Cancer 45: 893-898

26. Ziegler HW, Kay NE, Zarling JM (1981) Deficiency of natural killer activity in patients with chronic lymphocytic leukemia. Int J Cancer 27: 321-327 